\title{
Corrupción ¿calle de una sola vía? La internalización del soborno en empresas en México
}

\author{
Corruption, a one-way street? Internalization \\ of bribery in Mexican Companies
}

\author{
David Arellano Gault \\ Centro de Investigación y Docencia Económicas A.C. (CIDE), \\ Ciudad de México, México \\ ORCID: http://orcid.org/o0oo-0002-II56-3568
}

DoI: http://dx.doi.org/I0.28928/revistaiztapalapa/842018/aoti/arellanogaultd

\section{Resumen}

En un país que sufre de corrupción sistémica, la interacción entre empresas y servidores públicos suele estar permeada por diversos actos que pueden ir desde el soborno a la captura del Estado por actores privados poderosos. Es decir, la corrupción es una calle de doble vía donde gobierno y empresas se refuerzan mutuamente, incluso a través de acuerdos y negociaciones. Por medio de una encuesta a empresas privadas en una ciudad mexicana y luego de un conjunto de entrevistas a profundidad en dos de ellas, este artículo muestra cómo las empresas construyen rutinas y soluciones internas que les permitan atender la demanda de soborno por parte de los servidores públicos. En otras palabras, las empresas crean procesos internos estables y organizados para pagar los sobornos como un acto normalizado. A partir de un estudio empírico se aporta al debate en la literatura sobre corrupción que aduce que esta (en este caso, en su lógica de soborno), más que ser un acto aislado de individuos, es una relación social que tiende a normalizarse y racionalizarse. Estos hallazgos respaldan la discusión de que para entender el fenómeno de la corrupción es necesario vincular tanto las perspectivas de los individuos corruptos como aquella que la observa como un fenómeno social de intercambios, favores y reciprocidades.

Palabras claveः soborno, corrupción, administración pública, normalización de la corrupción

\footnotetext{
Abstract

In a country that suffers from systemic corruption, the interaction between companies and public servants is usually permeated by diverse acts that can go from bribery to the capture of the state by powerful private actors. That is, corruption is a two-way street where government and companies reinforce themselves. Through a survey of private companies in a Mexican city and after a series of in-depth interviews in two of them, this article shows how companies build routines and internal solutions that allow them to meet the demand of bribery from public officials. In other words, companies create stable and organized internal processes to pay bribes as a normalized act. Companies, facing requests for bribes, build an organizational process in which diverse internal actors participate and justify their actions, making these processes normal and stable routines for the company. These findings support the argument that in order to understand the phenomenon of corruption, it is necessary to link both the perspectives of the corrupt individuals and the one that observes it as a social phenomenon of exchanges, favors and reciprocities.

Keywords: Bribes, Corruption, Public sector and administration, Normalization of corruption
}

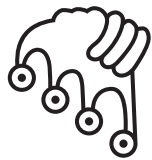

IZTAPALAPA

Agua sobre lajas

* david.arellano@cide.edu 


\section{Introducción}

حuando se discute el problema de la corrupción sistémica en un país se tiende a enfocar las acciones del sector público llegando a pensar que las empresas son básicamente víctimas. ${ }^{1}$ La corrupción se expresa en realidad en una multiplicidad de tipos de acciones, lo cual implica comprender la especificidad de cada tipo de corrupción y la lógica social que lo sustenta. Sin embargo, al admitir que algunos actos de corrupción comienzan en el sector público y atrapan de esa manera a las empresas (por ejemplo, muchos casos de soborno), quedan algunas preguntas organizacionales por contestar: ¿qué procesos internos se desencadenan en una empresa para acatar el soborno solicitado por uno o varios servidores públicos? ¿Esos procesos internos que desata la organización para responder al soborno, se estabilizan y racionalizan por decisión de sus miembros? ¿Qué tanto ese proceso de estabilización diluye las fronteras entre víctima y victimario, haciendo a la empresa incluso proactiva en el proceso de cumplimiento y sostenimiento de los acuerdos de soborno? Estas preguntas asumen la corrupción más como una relación social que como "calle de un solo sentido", donde uno de los actores es "el corrupto" y otro "la víctima".

La corrupción vista como relación social permite pensar que esta puede llegar a ser sistémica (Arellano, 20I2), es decir, que constituye en la práctica un entramado en el que se encuentran inmersos diversos actores sociales, tanto del sector público como del privado. La corrupción vista como proceso social implica que las personas terminan constituyendo una densa red de transacciones (muchas de las cuales pueden no ser corruptas) con vínculos estables que se reproducen y que pueden considerarse, según la perspectiva, como impropios o ilegales, pero también como actos relativamente normales o comunes, una especie de organización social de la corrupción (Mungiu-Pippidi, 2006:87). Este enfoque tiende a ser escéptico

1 En México ese sesgo ocurre con la Encuesta Nacional de Victimización de Empresas (eNVE) del INEgi (20I4a). 
ante la idea de que la corrupción es un acto de individuos aislados o de "manzanas podridas". Por el contrario, plantea que los diversos actos que puedan considerarse como corruptos son pate de una relación social bien constituida, muchas veces estable y que es capaz de autorreproducirse. La corrupción sistémica puede pensarse entonces como una trampa social clásica, ya que no es fácil para ningún actor salirse del "acuerdo" amplio que permite una relación con actos corruptos pero que han devenido consuetudinarios y constantes (Persson, Rothstein y Teorell, 2013).

Desde el enfoque de la corrupción como un sistema o una organización social, suponer que las empresas son solo víctimas de la corrupción proveniente del sector público puede estar perdiendo de vista la riqueza de los procesos sociales y políticos involucrados en actos como el soborno. Para analizar esta idea, el presente estudio se concentra en un tipo de corrupción común en muchos países: el soborno. En este caso, el que inicia con una solicitud de dinero a cambio de servicios o permisos (este puede ser un tipo de soborno distinto a aquel que puede ser iniciado por las empresas para obtener influencias que las beneficien). El punto está en investigar para conocer la dinámica interna y organizacional que una empresa dispara para atender la petición de soborno, para atenderla organizacionalmente y probablemente para hacerla predecible y estable en el tiempo. Las empresas, entonces, probablemente pueden ser vistas como víctimas en primera instancia, pero ante la necesidad de hacer estable el intercambio que sustenta el soborno, primero, requieren transformar cierta parte de su lógica interna para normalizar el intercambio. Y segundo, es admisible pensar que necesitan impulsar un proceso de estabilización interno a la empresa para hacer del soborno recurrente un acuerdo estable y predecible.

Para enfrentar una petición de soborno desde la empresa (que tiene, en sociedades de corrupción sistémica, una alta probabilidad de repetirse en el tiempo) se requiere crear y estabilizar un proceso, una serie de pasos racionales y organizados que involucran a diferentes personas o empleados a efectuar determinadas actividades con el fin de cumplir o satisfacer las demandas del soborno. Este proceso interno de respuesta al soborno puede ser costoso en diferentes formas. No implica sólo el pago del soborno, sino el proceso organizacional que debe dispararse para satisfacerlo. Y este proceso implica que tiene que ser justificado o explicado, o incluso escondido, a las propias personas de la organización que están involucradas. La cuestión es, justamente, comprender cómo estabilizar dicho proceso para la propia empresa se convierte en una necesidad racional. No hay que olvidar que la empresa obtendrá algo de la transacción: la licencia, el trámite o el permiso indispensable para trabajar o existir. En esa transacción, el soborno desata una relación que implica crear internamente las condiciones materiales y simbólicas para su realización efectiva. 
Es justamente este último punto el que está muy poco estudiado en la literatura, al menos de manera empírica (comprensible dada la dificultad de estudiar un proceso que suele ser oculto e incluso negado por las personas involucradas).

El objetivo de esta investigación es dilucidar empíricamente los procesos internos que adoptan las empresas para interactuar y cumplir con solicitudes de soborno iniciados por servidores públicos a cambio de entregar un permiso, una licencia o un trámite. La hipótesis central es que, en una situación de corrupción sistémica, las empresas pueden precisar hacer endógena la lógica del soborno con el fin de entrar en una relación fluida y con el menor costo posible con los servidores públicos corruptos. Es decir, las empresas crean procesos internos para decidir y negociar la relación con dichos servidores, conseguir los recursos y sustentarlos financieramente para crear las condiciones que permitan asegurar la estabilidad del trato en el largo plazo (proceso estudiado ampliamente a nivel teórico por Ashforth y Ananad, 2003; Ananad, Ashforth, Joshi y Martini, 2004; Kish-Gephart, Harrison y Klebe, 2010). Esto implica comprender a qué nivel las empresas se convierten en algún sentido en estabilizadoras, por necesidad y por interés propio, de la relación de soborno.

Un país con alta incidencia de corrupción como México es un caso sólido para estudiar el proceso que siguen las organizaciones para hacer endógenos los actos de soborno solicitados por servidores públicos corruptos. Dichos actos son consuetudinarios (de acuerdo con INEGI 20I4a, II.3 \% de los delitos que victimizaron a empresas en México fueron actos de corrupción ${ }^{2}$ ), se convierten en normales y requieren orden procedural para su ejecución estable.

La sistematización de la corrupción por organizaciones gubernamentales es algo bastante estudiado (Arellano, 2012; Scott, 1969; Rothstein y Teorell, 2008; Johnston, 2005; Heywood, 20I5, por mencionar unos cuantos). Pero no tanto su contraparte: el proceso a través del cual las empresas normalizan la interacción de soborno que solicita el servidor público. Este enfoque permite preguntarse qué tanto las peticiones de soborno son procesos de corrupción exógena (donde la empresa es una víctima) y qué tanto al normalizar estos actos se originan procesos endógenos en el seno de la empresa. Esta lógica implica pensar en una dinámica de corrupción normalizada, donde la compañía procura mantener estable la relación

2 De estos delitos, $44.9 \%$ implicaron a funcionarios encargados de otorgar permisos o licencias; $19.9 \%$ incluyó a policías, jueces y ministerios públicos; 19 \% a funcionarios del sector salud, tributario, de seguridad social o de protección civil; y el resto, a servidores de obra pública y de bienes y servicios (INEGI, 20I4b). Otros estudios basados en percepción indican también que México refleja un alto índice de soborno (Transparencia Internacional, 2017). 
de corrupción que le resulta más conveniente, convirtiéndose en un agente proactivo y partícipe del proceso o círculo de corrupción. Comprender ese proceso de estabilización puede ser de suma importancia tanto para comprender la dinámica real de los actos de corrupción como para entender detalles fundamentales del fenómeno con la finalidad de combatirlo más efectivamente.

Para estudiar el proceso de estabilización de la relación que dispara la solicitud de soborno se realizó un estudio con base en una encuesta a empresas medianas de una de las ciudades económicamente más importantes del país; y a partir de los resultados se realizaron estudios a profundidad (por medio de entrevistas) en dos de las empresas a partir de una lógica de contraste. Los resultados permiten comprender de manera inicial al menos el proceso interno que se construye para procesar el soborno y estabilizar los acuerdos, y dan una muestra del tipo de relación que construyen las empresas con los servidores públicos en estas situaciones de venalidad constante.

El artículo se organiza de la siguiente manera: en la segunda parte se revisa la literatura de normalización de la corrupción a escala organizacional con el fin de discutir los procesos de racionalización necesarios para dar estabilidad y sentido a diversos actos que pueden ser considerados ilegales o poco éticos. En la tercera se presentan los elementos metodológicos de la encuesta que se desarrolló y la forma en que se escogieron los estudios de caso. En la cuarta se discuten los resultados de la encuesta y en la quinta los de los estudios de caso por medio de entrevistas. Por último, se presentan las conclusiones.

\section{Normalización de la corrupciónł una calle de doble vía}

La literatura de normalización de la corrupción estipula que se trata de un proceso con tiempos, reglas y dinámicas, no un acto discreto de dos individuos aislados. Por lo tanto, llegar al hecho corrupto en sí implica un pasado que permitió la gestación y el desarrollo del acto corrupto. Esta suerte de historicidad, además, tiene un futuro: para repetir ese acto debe mantenerse un proceso de relaciones sociales que incrementen la probabilidad de que el hecho se reitere (Blundo, Olivier y Arifari; $2006,33)$. En consecuencia, la característica más antiintuitiva de la corrupción en las organizaciones es que el cohecho se hace normal. La literatura al respecto está llena de evidencias y referencias, por lo general, a gente que ha sido procesada por corrupción y que niega haber realizado algún acto ilegal o incluso inmoral (Benson, 1985; Conklin, 1977; Cressey, 1986; Geis y Meier, 1979; Sykes y Matza, 1957). 
Una explicación posible para esta aparente paradoja puede encontrarse en la capacidad de las organizaciones para rutinizar y normalizar los diferentes valores, actividades y objetivos de los individuos y los grupos que las integran. Del mismo modo como una organización normaliza el respeto por la jerarquía o los procedimientos aceptables en el trabajo diario, se pueden normalizar comportamientos clasificables como corruptos a partir de incrustarlos en los procesos y prácticas del día a día, socializándolos dentro de la organización como relativamente "normales". Incluso, puede ocurrir que un grupo de comportamientos no corruptos esté combinándose con otros que sí lo son, haciendo que un individuo particular en la empresa no tenga la "fotografía completa" del engranaje de actos que llevan a un resultado ilegal o corrupto. Esta lógica hace entonces posible comprender cómo una organización y muchas de las personas en ella caigan en la corrupción poco a poco, como en una resbaladilla (Arellano, 2017).

Las personas en las organizaciones requieren construir el sentido de lo que hacen y su perspectiva es siempre limitada (por tiempo, recursos, capacidad, como dice el concepto clásico de racionalidad limitada de Simon, 1947). En consecuencia, una organización construye una serie de mecanismos de influencia, rutinas y principios que ayudan a los miembros de la organización a dar sentido a sus acciones; ahorrando tiempos a través de rutinas, propiciando la cooperación a través de procesos estandarizados, especializados y compartimentados. Es mediante estos mecanismos de influencia, intencionalmente o no, como pueden construirse esquemas de acción normalizada y comportamientos corruptos.

La acción organizacional es una construcción social, según Berger y Luckmann (1978): los actores organizacionales construyen diferentes interpretaciones y lealtades para internalizar diferentes lógicas de socialización. El grupo, el subgrupo, el departamento, la organización como un todo; la industria donde la organización se mueve, y por último, el país, la sociedad, son diferentes niveles donde el actor internaliza y socializa las normas, las reglas, las expectativas, incluso los principios morales que le darán cabida en dicha colectividad (Chibnall y Sauders, 1977, I4I). Evidentemente, esa socialización secundaria, como le llaman Berger y Luckmann, implica un proceso que puede ser contradictorio al interpretarse en diferentes niveles de agregación.

En corto, las lógicas de socialización de uno de los estratos (por ejemplo, la organización) pueden chocar con otro (por ejemplo, el grupo). Pagar sobornos - como es el centro de análisis del presente estudio - puede ser lo adecuado para una empresa que se considera víctima de servidores públicos corruptos, pero altamente estresante para el miembro de la compañía que está encargado de negociar 
y pagar el soborno. Por ejemplo, maquillar algunas cifras financieras puede ser benéfico para la organización, bajo el argumento de que buena gente se equivocó con buenas intenciones; pero visto como un acto de corrupción a nivel de la sociedad puede resultar en tensiones al contraponer las lógicas de distintos estratos de socialización. Para lidiar con estas contradicciones fundamentales la respuesta parece ser la racionalización.

La racionalización es un acto de interpretación e indispensable para que una persona pueda ser un actor social. Al menos desde Goffman (198I), se comprende que los individuos saben presentarse ante los demás con una estrategia fundamental de claroscuros; que permitan construir una imagen determinada y dar cierta certidumbre a la interacción esperada con los demás. ¿Por qué se obedece a un jefe? Porque se cree en la legitimidad de su jerarquía, conforme a Weber (1922/2002). Eso es una racionalización necesaria para la constitución del tejido de interacción social. Es una racionalización que no deja de ser una interpretación, una estrategia de sentido, una amalgama de esencia y apariencia de aquello que los actores desean se observe y de lo que desean permanezca oculto - la opacidad es un ingrediente fundamental en la interacción (Costas y Grey, 2014) - . No solo la opacidad, sino el secreto y la secrecía forman parte inevitable del repertorio de estrategias de interacción y comunicación en las organizaciones (Zerubavel, 2006).

Cuando se trata de corrupción, los actores dentro de la organización pueden haber creado una racionalización que permite construir ese acto como un accidente o como necesario para lograr un bien común mayor. Esta racionalización busca mantener cierto nivel de salud moral colectiva que justifique al grupo (Ashforth $y$ Anand, 2003: 16). Incluso es posible crear eufemismos para referir ese acto corrupto.

El abanico de opciones de racionalización organizacional en casos de corrupción es diverso y amplio: negación de la responsabilidad ("me fue ordenado"), negación del daño ("es un soborno que no afecta a nadie por su tamaño o por los efectos positivos que traerá para la empresa"), negación de las víctimas ("los corruptos son los otros, yo solo pago el soborno dado que sería más costoso no hacerlo") y el respeto a lealtades más elevadas ("pagar un soborno puede ser un acto corrupto, pero solo así aseguro la supervivencia de mi negocio o de mi empleo") (Ananad et al., 2004; Ashforth y Ananad, 2003; Ashforth et al., 2008; Felps, Mitchell y Byington, 2006; Fleming y Zyglidopoulos, 2009; Kish-Gephart et al., 2010; Pinto, Leana y Pil, 2008).

Evidentemente, estas racionalizaciones pueden ser interpretadas como simples mentiras o justificaciones hipócritas. Sin embargo, la historia no parece ser tan simple. Muchas veces, estas racionalizaciones son verdaderos mecanismos de interpretación y justificación que permiten al actor sentirse mejor consigo mismo, 
reduciendo la angustia o la disonancia entre sus valores generales y sus actos particulares (Gigerenzer, 2002). El autoengaño en psicología no es exclusivamente de hipocresía, sino un proceso mental y social de justificación y modificación de los parámetros de interpretación por parte de los seres humanos (Lerner y Tetlock, 1999: 263; Bargh Tanya y Chartrand, 1999: 475).

El autoengaño, con todo lo antiintuitivo que suena, es estudiado ampliamente en la psicología como un acto de modificación de los parámetros mentales para evaluar la realidad (Ainslie, 200I). En este sentido, las personas construyen historias y percepciones de tal manera que sean capaces de reducir, o incluso eliminar (al menos temporalmente) la disonancia cognitiva que les produce saber que están cometiendo un acto indebido (Festinger, 1957). Dicha disonancia es una tensión interna de las ideas o emociones de la persona con respecto a lo que observa que es o puede hacer de la realidad. Al reducir la disonancia, es posible sentirse menos afectado moralmente.

Una persona puede reducir la disonancia cognitiva si racionaliza que el acto de corrupción no fue tan grave, que fue seguido por otros, ordenado por otros, o si forma parte de un acto de "justicia" redistributiva contra los "verdaderos actores perversos". El punto central es que la racionalización implica un esfuerzo real, creativo y con impactos efectivos en la interpretación de la persona respecto al grado de honestidad de un acto. Adicionalmente, esta racionalización puede estar reforzada por la organización y sus grupos, de forma sistemática o implícita en las rutinas, prácticas y eufemismos existentes.

La racionalización es un paso fundamental en el contexto que permite a las personas enfrentar $y$ justificar sus actos aun cuando saben, o intuyen al menos, que estos alimentan o forman parte de una cadena de corrupción. Ahora, si a la capacidad de racionalización le añadimos que las organizaciones van creando procesos y rutinas, adquiere más sentido la idea de que las personas y las organizaciones caen poco a poco en una resbaladilla de corrupción.

El proceso mental y relacional que lentamente racionaliza el tamiz de un acto corrupto puede ser reforzado ampliamente por la dinámica organizacional. Precisamente, cuando una persona ingresa en una organización inicia un denso proceso de socialización y decodificación: comienza a aprender qué es la organización, quiénes la componen, qué grupos la manejan, cuáles son los valores imperantes de jerarquía, obediencia, cooperación, conflicto y negociación (Kunda, 1992). Estas dinámicas sociales permiten a las personas ser introducidas, aprender, construir su rol y su papel; funcionan para cuestiones formalizadas (como identificar la jerarquía y las reglas de comunicación), así como para la socialización de actos o comportamien- 
tos que puedan, en el tiempo, alimentar la corrupción. Es importante comprender esta contradicción, dado que explica por qué, en general, las personas acusadas de corrupción no se asumen como culpables de dichos actos. No se trata solo de cinismo o de cálculo para negar la corrupción. Tampoco solo del proceso psicológico que permite la racionalización.

En realidad, el tema es una mezcla en la cual los propios procesos organizacionales permiten a una persona ser parte del grupo, de la organización; de aquellos procesos que actúan y funcionan para ubicar y posicionar al actor en tramas de relaciones y actores que, al final de cuentas, pueden ser clasificados como corruptos. Empero, en este caso, son actos corruptos que los propios procesos organizacionales, paradójicamente, ayudaron a cimentar, consolidar y hacerlos consuetudinarios.

La dinámica social de introducción de una persona a la lógica organizacional se da poco a poco, como un proceso de aprendizaje de las reglas y las normas de interacción en un colectivo. La metáfora de cómo los procesos mentales y sociales de racionalización pasan a ser reconstruidos y fortalecidos por la lógica organizacional es el de la "resbaladilla".

En el caso de la corrupción, las organizaciones pueden crear condiciones para que las personas "resbalen" paulatinamente a cometer actos corruptos en un proceso facilitado, en el que dichos actos sean racionalizados y justificados como "normales" o, al menos, aceptables desde la lógica del grupo o la organización. Para que el efecto "resbaladilla" ocurra, es necesario que la persona incorpore las reglas y prácticas organizacionales, aceptándose como un agente incrustado en tales prácticas. Con este proceso, la persona en la organización sigue siendo aquel individuo que ingresó en ella, pero a la vez es otro: el que obedece, es aceptado, "sabe" interpretar y comprender la "cultura" o la "naturaleza" de la organización, como un submundo donde muchos de sus comportamientos y sentimientos solo adquieren lógica mientras está dentro de la organización.

Las dinámicas de racionalización y socialización se refuerzan mutuamente y crean una especie de lógica de endogenización donde las personas y las mismas organizaciones se apropian del discurso y de las prácticas para justificarlas. Cuando se deja de lado el supuesto útil — pero demasiado simple — del decisor individual calculador que decide ser corrupto; cuando se comprenden los mecanismos mentales y sicológicos para racionalizar $y$ dar sentido a los actos propios; y se introduce una arena de relaciones, reglas y tiempos organizacionales, la corrupción comienza a ser vista desde una perspectiva más dinámica. Pero también más preocupante, pues puede intuirse la dificultad que tienen los mecanismos anticorrupción para lidiar 
con un fenómeno que se construye organizacional y socialmente en las prácticas y racionalizaciones del día a día de las personas.

\section{Estudio de campo: encuesta y análisis de casos de contraste}

Para estudiar entonces el proceso a través del cual las empresas hacen endógeno el soborno en situaciones de corrupción sistémica se efectuaron dos estudios relacionados: una encuesta y un estudio de dos casos contrastantes. Tomando a México, un país de altos niveles de corrupción, se eligió una ciudad de las primeras io del país en actividad económica con el fin de asegurar el análisis de situaciones de relación empresa-gobierno de alta importancia. Además, se consideró sustancial que esa ciudad fuera seleccionada también por la alta incidencia de corrupción. ${ }^{3}$ En este caso se optó por una que ocupa aproximadamente $16 \%$ del empleo total nacional en cerca de 500000 empresas en su territorio. Se seleccionó como muestra para la encuesta a empresas de un tamaño económico importante sin que pudieran ser consideradas muy grandes. Esto con el fin de ubicar el objeto de estudio en compañías que por un lado tienen ya un tiempo importante de existencia y por otro que no supusieran necesariamente una alta capacidad de captura del gobierno como podrían ser el caso de las empresas muy grandes (llevando a otro tipo de corrupción como la colusión para obtener contratos gubernamentales con condiciones preferenciales). Para ello se seleccionaron aquellas que se pudieran considerarse en el rango alto de las llamadas empresas medianas (INEGI, 20I4C): de entre IOI y 250 empleados y un rango anual de ventas de IOO a IIOO millones de pesos mexicanos (5.5 a 59.5 millones de dólares). Además de este criterio para la muestra se tomaron los sectores de la actividad económica con mayor probabilidad de requerir permisos, licencias o autorizaciones para funcionar de manera regular (sectores agrícola, industrial y comercio, dejando fuera por tanto el de servicios). La encuesta enviada solicitó información a las direcciones generales de las empresas con respecto a cómo son victimizadas por servidores públicos de la ciudad para obtener permisos o licencias críticas para su existencia.

3 México es el lugar 95 de 167 países evaluados en TI (2015); y la ciudad seleccionada está en el más alto rango de percepción de corrupción ( $88.5 \%$ de su población mayor a I 8 años) de entre las primeras io ciudades en términos de actividad económica en el país (INEGI, 2OI4b). 
Bajo estos criterios se obtiene un universo muestral de i 156 empresas en la ciudad. Se envió un cuestionario ${ }^{4}$ por correo electrónico a los directivos generales, con el fin de que respondieran por ese medio. Se dio seguimiento mediante llamadas telefónicas y dos mensajes de recordatorio. El cuestionario constó de I9 preguntas con opciones semicerradas. El volumen de respuesta fue de 389 cuestionarios atendidos, pero solo 306 completos. Estos últimos se consideraron válidos, de modo que la tasa de respuesta fue de $26.4 \%$, lo que es una muestra relativamente aceptable para un estudio sobre un tema tan intrincado como el del soborno, y consideramos que permite con cierta confianza plantear, con precauciones necesarias, algún nivel de generalización sobre el proceso de internalización del soborno en las empresas en México.

El cuestionario establece de inicio que el objetivo de la encuesta es comprender cómo la empresa se ve obligada a acatar solicitudes de soborno por parte de servidores públicos, aclarando que - de acuerdo a la legislación nacional existente en ese momento en el país-, la empresa y las personas que en ella laboran no están cometiendo un delito si aceptan pagar el soborno. La ley mexicana, al momento del estudio, establecía que el delito se cometería por parte de la empresa si la misma, de manera espontánea, ofrece el soborno (art. 222 del Código Penal Federal). En esta encuesta siempre se asume que la iniciativa de soborno es por instancia del servidor público, y se le informa de ello al empresario.

Una vez procesadas las encuestas, se seleccionaron dos casos contrastantes para estudiarlos en mayor profundidad. ${ }^{5} \mathrm{El}$ contraste estaría entre un caso donde la empresa plantea que es fundamental mantener la estabilidad de los acuerdos que

4 Para la realización de esta encuesta se construyó un protocolo de seguridad y anonimato que fue discutido con diversos empresarios, quienes fungieron luego como comunicadores de la confiabilidad que otros empresarios de la ciudad podrían tener en la realización de este estudio. Este proceso de creación de confiabilidad fue indispensable para lograr el nivel de respuesta que se alcanzó. Derivado de estos acuerdos, la presentación de datos y los detalles del estudio se mantienen en un alto nivel de generalidad con el fin de evitar en la medida de los posible la identificación de la ciudad y de las empresas en el estudio.

5 Debido al mismo protocolo de seguridad antes mencionado, las entrevistas no pudieron ser grabadas. Sólo se permitió al investigador atender a las entrevistas (sin auxiliares, por ejemplo) y apuntar en un cuaderno lo que se iba discutiendo en tiempo real. Esto sin duda es un límite importante de la presente investigación, aunque es un costo que por el tema de estudio y lo delicado de la cuestión que trata, se consideró como aceptable para, sin embargo, realizar interpretaciones plausibles y con un grado básico pero existente de validez externa para los fines del estudio. 
posibilitan el soborno de manera sistemática (6o empresas así lo consideraron. Véase más adelante la encuesta), y otro donde mantener esa estabilidad del acuerdo es considerado de importancia mediana o poca (65 empresas lo consideraron de esa manera). Adicionalmente, se buscó que los casos fueran empresas que asumen el soborno como proveniente de una red de servidores públicos y no de un servidor público aislado. Esto con el fin de maximizar el grado de dificultad, desde la perspectiva de la empresa, de salirse del acuerdo y la necesidad de estabilizarlo. De estos casos identificados, I2 empresas que cumplían con estos criterios contestaron estar dispuestas a ser estudiadas mediante de una entrevista presencial (siete empresas que consideraban importante realizar esfuerzos de estabilización y cinco que no). De estas se escogieron dos empresas con lógicas contrastantes en el sentido antes dicho. Las entrevistas duraron en total aproximadamente cinco horas, dado que se solicitó interrogar a más de una persona conocedora de la situación (tanto directivos como gerentes): cinco personas en el caso de la empresa que consideró muy importante mantener estables los acuerdos, y seis en la empresa que lo consideró no tan importante).

\section{Resultados de la encuesta}

El cuestionario constó de 19 preguntas, ${ }^{6}$ todas referidas a la experiencia de la empresa en 2015. El primer bloque buscó comprender la visión de la corrupción en el sector público e indagar si la empresa recibió solicitudes de soborno. El 84.6\% de los encuestados consideró que la autoridad de la ciudad es corrupta, y $90.8 \%$ indicó que ha sufrido al menos una solicitud de soborno en el año referido. El promedio de incidencia de sobornos fue de 3.7 veces en el periodo estudiado. De 278 empresas que respondieron haber sufrido de soborno en el año, $26.6 \%$ señaló que el monto de dinero solicitado fue de entre 5001.00 y 25000.00 pesos (aproximadamente entre 270.00 y I 345.00 dólares); 4I.7 \% reportó que el monto ascendió a una cantidad de entre 25 00I.00 y 125000.00 pesos (entre I 350.00 y 6753.00 dólares); y I2.2 \% señaló que el soborno fue de más de 125 00I.00 pesos (más de 6753.00 dólares). Por último, 6.I \% reportó haber pagado el soborno en especie, es decir, en otra manera diferente al dinero. De estos, 58.8 \% refirió haber otorgado regalos "materiales", y 4I \% pagó en regalos y "servicios".

6 Véase el anexo I con una versión simplificada del cuestionario. 
Respecto al proceso interno que la empresa articula para tratar el soborno, $24.4 \%$ de las empresas que lo sufrieron reportaron que lo contabilizan como un gasto especial, y $56.4 \%$ señaló que lo identifican como "otros gastos". El 17.6 \% reportó que internamente no tienen una definición contable para procesar el soborno. En cuanto a cómo la empresa genera un proceso interno para tratar la solicitud de soborno, $56.4 \%$ reportó que el caso lo llevan de una a dos personas; $26.2 \%$ de tres a nueve personas; y $17.2 \%$ señala que es tratado por Io o más personas. Al respecto, es interesante observar que solo $23.7 \%$ reportó que la totalidad de las personas involucradas en el procesamiento del soborno saben que, en efecto, están tratando un proceso organizacional para desahogar el soborno. Del grupo de entrevistado, $39.5 \%$ planteó que, aproximadamente, tres cuartos de los involucrados en el procesamiento del gasto del soborno sabía que estaba procesando el soborno; y $36.6 \%$ afirmó que la mitad o menos de las personas involucradas tenían conocimiento; $74.8 \%$ considera que el soborno le es solicitado por una red de servidores públicos y solo I $4.3 \%$ cree que es un individuo el que lo solicita. En cuanto al lugar donde se establecen los acuerdos, $42.8 \%$ reportó que se realizan en una comida o desayuno fuera de la empresa, 35.9 \% en la empresa misma, y i6.I \% señaló que ocurre en las oficinas del servidor público.

El cuestionario también indaga acerca de cómo es la relación con el servidor público que solicita el soborno; $32.01 \%$ reporta que este ha tratado de cambiar las reglas del juego para dificultar la legalidad de la empresa. Ello genera un problema para la firma, tres de cada cuatro (75.5\%) consideró la estabilidad de los acuerdos como un problema "importante" o "medianamente importante". En consecuencia, $82.9 \%$ indicó que realiza algunas actividades para "mantener" el buen trato con el servidor público (invitaciones a actividades informales, regalos). No obstante, $38.84 \%$ también admitió que utiliza amenazas o negociaciones fuertes para mantener estable el convenio del soborno. Incluso uno de cada io (II.7 \%) ha pasado de las palabras a los hechos y denunció formalmente los actos de corrupción en algún momento. Empero, resulta interesante que $60.1 \%$ contestó que no ha realizado tal demanda; y $24.18 \%$ prefirió no responder a la pregunta. Las razones para que las empresas se atrevieran a demandar fueron debido a problemas económicos de la empresa (44.44\%), abusos del servidor público (II.I \%), y por cambio de la administración en el gobierno (22.2\%) -lo que presumiblemente generó cambios bruscos en el trato establecido con anterioridad-. Por último, ante la pregunta de si el soborno y lo que tiene que hacer la empresa para entregarlo ha afectado negativa o positivamente a la empresa, tres de cada io dice que la empresa ha cambiado para mal, 
mientras que $7.5 \%$ opina que para bien, y $47.4 \%$ considera que no la ha afectado ni positiva ni negativamente.

\section{Resultados de los estudios de caso}

Como se explicó previamente, se realizó un estudio en profundidad de dos casos contrastantes. Dos empresas que respondieron distinto a la pregunta: ¿Qué tan importante es para su supervivencia y la de negocio el mantener estables los acuerdos - previamente establecidos - para procesar exitosamente el soborno solicitado por un servidor público? De acuerdo con la selección, se entrevistó a directivos de dos empresas, una que planteó que era importante mantener estables esos acuerdos, y otra que no lo consideraba tan relevante. Puesto que la investigación pretende comprender el proceso interno que organizacionalmente se desata por sobornos en situaciones de corrupción sistémica, la lógica indicaría que la estabilización de los acuerdos es fundamental, de ahí la importancia de contrastar ambos casos.

Para efectos prácticos, los casos seleccionados los denominaremos como Empresa pro estabilidad y Empresa indiferente. La idea central de las entrevistas semiestructuradas fue comprender el proceso organizacional interno que la empresa articula para enfrentar la solicitud de soborno. Sobre todo, en términos de la normalización de procesos estándar que la empresa propicia en sus rutinas. Las entrevistas se practicaron a directores generales y directores de finanzas de cada empresa. La entrevista siguió un guion semiestructurado con el objeto explícito $-\mathrm{y}$ acordado con los entrevistados - de conocer más detalles sobre su experiencia al enfrentar el soborno con servidores públicos (tomando diversas precauciones para asegurar la confidencialidad de los datos de la empresa y de las personas entrevistadas).

\section{Empresa pro estabilidad}

De acuerdo con los directivos de esta empresa, el soborno es una petición constante por parte de servidores públicos de la ciudad, quienes aprueban licencias o tienen la facultad de dar permisos diversos sin los cuales la empresa no podría operar. Esta compañía en particular existe desde hace más de 30 años, y ha visto desfilar gobiernos de la ciudad pertenecientes a diferentes partidos políticos, sin que se note una diferencia sustantiva en cuanto a mayor o menor proclividad a solicitar sobornos. Cuando hay cambios de partido gobernante se deben desarrollar nuevos códigos y otras formas de negociación, pero las solicitudes de soborno son una constante. 
En palabras de un entrevistado: cuando hay cambios de partido gobernante "se requiere un esfuerzo nuevo por redefinir las reglas del juego". En la práctica puede significar varios meses de renegociación con las modalidades, cantidades y modus operandi cambiantes. Sin embargo, una vez establecidas las nuevas reglas, los tratos de soborno se han mantenido relativamente estables.

Los empresarios entrevistados dejaron claro que no hay margen de maniobra: "intentar oponerse a la corrupción es sumamente costoso y puede que hasta peligroso". La empresa contabiliza de manera sistemática los pagos, los tiene incorporados en los costos de operación. Cuando un servidor público intenta cambiar las reglas, la empresa ya tiene maneras de saberlo, pues muchas veces son amenazas "entendibles" dentro del proceso de negociación. $\mathrm{Al}$ respecto, los entrevistados no desearon abundar mucho, pero dejaron claro que no solo es cuestión de dinero: "se establece en general una relación constante de favores y servicios para mantener estabilidad en la relación con los servidores [públicos]". De acuerdo con estos directivos, "es lo más inteligente a hacer... es una inversión al final de cuentas... desarrollar lazos cercanos a la amistad con los servidores públicos a través de favores y de cumplir los acuerdos puede ser estratégico para la empresa”."Hacer honor" fue la nomenclatura que se usó, aunque fuera paradójico y conceptualmente extraño hablar de bacer honor en un intercambio basado en una solicitud de soborno.

\section{Empresa indiferente}

Esta empresa existe en la ciudad desde hace I4 años. Planteó que no es muy importante procurar la estabilidad de los acuerdos de soborno en el tiempo. Durante las entrevistas, la lógica básica parece similar a la de la empresa pro estabilidad: "las peticiones de soborno son constantes”. Sin embargo, en este caso el vocero señaló que desde su surgimiento como empresa enfrentó un sistema altamente desarrollado e institucionalizado de soborno. Es decir, "no fue necesario o importante hacer mucha negociación", dado que las cuotas, los pagos y las peticiones de dinero para obtener permisos o licencias estaban claramente especificados, eran sistemáticos y era prácticamente una rutina organizada desde el propio sector público. Si bien reconoció que existe la necesidad de establecer ciertos lazos directos con las personas del servicio público encargado del trato ilegal, los procesos están organizados y son estables. Cuando alguno de los procesos (ya sea la cantidad de dinero o la manera de entregarlo) se va a modificar, se le avisa a la empresa con tiempo suficiente, de modo que no sea una sorpresa que requiera cambios radicales o que desestabilice los costos de la compañía. 
Dada la sorpresiva institucionalización informal del sistema de soborno, la entrevista se salió del guion al preguntar a los empresarios que, dada su experiencia, ¿qué diferencia habría entre este sistema de corrupción desde el gobierno y uno de una mafia que ofreciera protección a cambio de dinero ("cobro de piso" es como se le llama a esta práctica que ejercen las mafias en México)? En primera instancia, los entrevistados se sorprendieron de la analogía, pero luego de reflexionarlo la respuesta fue clara: "en realidad ninguna diferencia". La empresa ha tomado estos pagos y esta lógica como parte normal y necesaria del proceso de existir en el mercado.

Ante la pregunta de si estarían dispuestos a denunciar estos actos de corrupción para tratar de obtener los servicios legítimos que les corresponden como contribuyentes, la respuesta fue que eso sería lo deseable. Empero, el director general puntualizó que tal escenario "si bien deseable en términos morales, en términos prácticos no tiene sentido: suponiendo que esta empresa denunciara, sabemos que hay otras muchas empresas que están tranquilas con este trato y con esta estabilidad, por lo que las consecuencias negativas y los costos serían solo para mi empresa". Por lo tanto, serían altos los costos de romper una dinámica de juego estable, pero ilegal. "No es racional [dijo] denunciar... seguramente me saldría más caro, y los resultados serían muy inciertos; y tal vez riesgosos —añadió con una sonrisa nerviosa”.

\section{Discusión}

Tomando en consideración el proceso a través del cual se seleccionó la ciudad, los sectores económicos y la muestra, así como el nivel de respuesta obtenido, podemos, con precaución, pensar que lo observado en este estudio es probablemente un indicio cercano de lo que pueda estar pasando en el resto de las relaciones entre empresas y gobierno en México. Evidentemente, como en cualquier estudio empírico, la interpretación debe realizarse con reservas. En este caso hablamos de indicios, no de generalización, más cuando tomamos en cuenta el impacto que pudo tener el estricto protocolo de seguridad y anonimato que el estudio requirió para ser viable. Por otro lado, en vista de lo delicado del tema, es posible decir que dicho protocolo valió la pena para tener información empírica de este tipo probablemente por vez primera, al menos en México.

Con ello en mente, podemos afirmar que, según los hallazgos, cuando la situación es de corrupción sistémica, las empresas construyen procesos estables y relativamente formalizados para articular la respuesta a las solicitudes constantes de soborno que reciben de funcionarios gubernamentales. Estos procesos se establecen de manera prácticamente rutinaria, buscando formalizar, en la medida de lo posible, un acto 
ilegal (por paradójico que parezca). $\mathrm{Al}$ respecto, se observa aquello que la literatura de corrupción organizacional ha planteado desde hace tiempo: con el fin de normalizar un acto corrupto sistemático, se crean procesos de racionalización que reducen la disonancia cognitiva que estos hechos podrían producir. En este caso, a las empresas les parece necesario para estabilizar estos procesos, pues una proporción relevante de estas coincide ( $75.5 \%)$ en que dicha estabilidad es "muy importante" o al menos "medianamente importante". Lo anterior implica asegurar que los tratos de soborno lleguen a ser algo constante, con reglas que se respeten y que permitan planificar y prever las necesidades de recursos. Es interesante la aparente paradoja: se espera que un acto ilegal como el soborno ocurra en una dinámica social estable entre los actores coludidos y, sobre todo, que sea honesta en el sentido de respetar los arreglos previos.

Estos resultados parecen apoyar la discusión que asume la corrupción no como un acto discreto, sino que es una relación social que tiene historia, que es producto de un proceso, y que puede esperarse se sostenga en el futuro. Una relación social que construye sus propios códigos, incluso códigos de respeto y honor (siguiendo el concepto de práctica de Bourdieu, 20I4): es importante sostener los acuerdos, y respetarlos una vez que se han establecido; aunque en el fondo los actores sepan que implican actos ilegales. Este proceso recurrente permite una lógica de normalización de la corrupción en la cual los encargados de procesar los sobornos puedan observarlos cada vez más como algo común. Este planteamiento parece sostenido por la perspectiva de la empresa indiferente ante un gobierno chantajista, pero estable y formal que le permite seguir compitiendo y viviendo en el mercado aparentemente sin mayores problemas. Por último, cabe señalar que la estabilidad de estos procesos internos y los acuerdos con los servidores públicos corruptos se convierten para los directivos de las empresas en factores sustantivos por alcanzar. A la llegada de nuevos servidores públicos, las dos empresas entrevistadas se confesaron proactivas con el objeto de rápidamente llevar a los servidores públicos a comprender las ventajas de los esquemas y procesos ya establecidos, versus las desavenencias que podrían emerger al cambiar la dinámica del juego.

\section{Conclusiones}

Este estudio es coherente con un grupo importante de discusiones clásicas que se han debatido en los últimos años, con respecto a las diferentes perspectivas que representa el fenómeno de la corrupción. En un extremo están las individualistas 
que comprenden el fenómeno como una decisión de cálculos de ganancias y riesgos; en el otro extremo está la corrupción definida como una relación social que se normaliza e institucionaliza. En este sentido, esta investigación provee pistas para discutir elementos desde ambas visiones.

Con respecto a la primera perspectiva, este estudio parece mostrar que actuar en pos de un soborno involucra, en efecto, cálculos de costo-beneficio. En este caso, el cálculo del sobornado es un mecanismo necesario para establecer de modo preciso las reglas del intercambio con el fin de obtener el permiso o la licencia — vitales para continuar como empresa-, y garantizar que el negocio siga siendo rentable. De acuerdo con los entrevistados, es más costoso negarse, implica costos de enfrentar juicios o demandas. Además, como problema de acción colectiva tiene costos vinculados a lo que otras empresas deciden estratégicamente: si una empresa no acepta el soborno, pero otras lo siguen haciendo, los costos se hacen inaceptables para la que decide mostrarse "honesta". En este sentido, es claro que la corrupción implica un cálculo, pero que depende de los actores, de su interrelación y de los efectos buscados en ambas partes por dicha interacción. El proceso mental y ético que esta interacción dispara en las personas es todavía poco entendido empíricamente, pero la idea de que en ciertas dinámicas las relaciones entre gobierno y empresas se "gansterizan" puede ser una pista a seguir (Fisman y Miguel, 2008; Gambetta, I993). "Gansterizar" implica una transacción que tiene sentido económico y social: las empresas y los servidores públicos entran en un mercado de "protección": protección contra daños y protección de otros competidores. Protección que, en otras palabras, adquiere sentido económico y estabilidad racional.

Entonces, estudiar el soborno como una forma de corrupción implica para la empresa hacer cálculos económicos y sociales; no es un actor pasivo que "lea" las intenciones $y$ tampoco necesariamente es un actor victimado unilateralmente (aunque esto puede ser verdad en ciertos momentos de la interacción). La empresa interactúa con los servidores públicos que le piden el soborno y se ve impelida a construir internamente, como organización, la respuesta y la racionalización de dicho soborno y de su operación.

Ahora bien, este estudio también abona al análisis de la corrupción como una relación social a través de un procesamiento organizacional endógeno. Los empresarios involucrados se formulan diversas preguntas que es necesario construir en términos sociales y organizacionales: $₫ \mathrm{El}$ solicitante del soborno es un individuo o es una red? ¿Hay muchos o pocos servidores coludidos? ¿Está organizado el soborno desde el propio gobierno? ¿Hasta qué nivel de autoridad participan los implicados en el soborno? ¿Qué riesgos hay si no acepto las condiciones? Además, 
se pregunta por otros actores: ¿Otras empresas están aceptando el soborno? ¿Eso debilita mi posición si yo decido demandar? Estas preguntas relacionales se pueden acompañar de otras más situacionales: en concreto, este servidor público que me pide el soborno, ¿̇en qué condiciones de negociación y regateo se quiere manejar? ¿Vale la pena generar vínculos más permanentes con él y la red de corrupción de la que es vocero? ¿Se puede confiar en que el funcionario hará honor (por paradójico que suene en estas circunstancias) al acuerdo? ¿Debo explicar a las personas de la empresa que están procesando un soborno o es preferible que no lo sepan?

La corrupción tiene una lógica que complejiza el cálculo: las relaciones, sus impactos, los símbolos, los lenguajes, el tempo, aparecen como elementos de un proceso social que tiende a construir los puentes para asegurar que la reciprocidad sea alcanzable y haya certidumbre: que el soborno se pida, se procese y se entregue. Diversas prácticas se van consolidando: hay maneras de acercarse al servidor público, hay formas de procesamiento, hay señales e interacciones para estabilizar los acuerdos; $y$ hay prácticas para incrementar la probabilidad de que el acuerdo se cumplirá. Las prácticas de la corrupción son lógicas, racionales: tienen sentido, logran objetivos para los actores involucrados y se mueven en una dinámica social relativamente estable y comprendida por los mismos actores - incluso bajo códigos y eufemismos-.

Este estudio entonces parece aportar también a las visiones que han definido la corrupción como un proceso social que depende en gran parte de la forma en que en una sociedad se ha negociado la lógica de la separación entre lo público y lo privado, entre lo debido y lo indebido (Bratsis, 2003). Dichas definiciones, que son críticas para comprender la corrupción, resultan fundamentales para definir lo que termina siendo socialmente "inaceptable". En los casos estudiados en este artículo tenemos un escenario contradictorio: la ley mexicana establece castigos y prohibiciones al soborno; pero, en la práctica, desde el punto de vista de la empresa, es adecuado, racional e inteligente procesar los sobornos para obtener los trámites o licencias para poder trabajar. Puede ser que los sobornos sean ilegales e incluso injustos. Pero si se estabilizan, si facilitan llegar a acuerdos con actores que se comportan paradójicamente- "de modo honesto" a la hora de cumplir los acuerdos, entonces la relación del soborno se racionaliza y cobra sentido. Incluso se normaliza, pues la organización crea las prácticas y rutinas para estabilizar la respuesta al soborno de la manera más económica y racional posible. Cambiar esta circunstancia puede ser muy costoso, un dilema de acción colectiva clásico: ¿Quién es el que iniciaría una revuelta contra los sobornos? En caso de que haya una empresa valiente, si se queda sola, puede que cargue con costos muy altos y resulte que, al final, la situación no 
cambió (y que quienes continuaron con el acuerdo de sobornos sí se beneficiaron y no pagaron costos).

Por otra parte, una de las limitaciones de este artículo es que no se conoce la postura de los servidores públicos que solicitan el soborno. Pero a partir de la información obtenida del caso de la "empresa indiferente" queda claro que detrás del servidor público sobornador también puede haber un proceso organizacional ordenado y rutinizado para procesar el soborno. Autores como Bordieu (20I4ः392393), precisamente, escriben de la hipocresía institucional: los servidores públicos, en esta esquizofrenia de separar radicalmente las esferas de lo privado y lo público, están en una situación de ambigüedad eterna. Un ejemplo: las reglas se pueden aplicar con absoluto rigor o con laxitud. Ninguna organización puede vivir si sus miembros aplican la regla al pie de la letra -_de ahí la forma de huelga conocida como "workto-rule" (Houba y Bolt, 2002) - . Las reglas en la acción organizacional se tienen que adaptar, que "doblar" (como la literatura de "bending the rules" ha mostrado, Sekerka y Zolin, 2007; Loyens, 20I4). Así, el servidor público comprende cómo, en la práctica, puede ser rígido o permisivo, ultrarrigorista o ultralaxista (Bourdieu, 20I4: 395). Este escenario de posibilidades genera una multiplicidad de estrategias para interactuar con las empresas o los ciudadanos. De ahí que, bajo esta lógica de interacción, puede interpretarse que la corrupción parece siempre un resultado lógico (y no una excepción) de la burocracia formal (Nuijten 2003, Shore y Haller, 2005:5): las reglas existen, pero son violadas constantemente ante la imposibilidad de comprender o actuar lógicamente ante la ilusión de una autoridad imparcial y una sociedad civil dispuesta a exigir esa imparcialidad. El soborno probablemente también requiere un proceso de normalización dentro de la organización gubernamental. Realizar dicho estudio es uno de los pendientes, sin lugar a dudas, aunque será sumamente difícil de realizarlo, por razones evidentes.

Finalmente, el presente estudio permite reflexionar sobre la corrupción como un fenómeno social denso: lógicas de reciprocidad e intercambio fundamentan socialmente el soborno, creando un entramado de favores, vínculos y expectativas entre empresarios y servidores públicos. Entramado que define la forma real del día a día de la interacción entre estos dos grupos de agentes sociales.

En países como México, entonces, es factible decir empíricamente que la corrupción (al menos esta vertiente de soborno que estudiamos aquí) se manifiesta a través de actos bien organizados e institucionalizados en las relaciones sociales del día a día. Organizacionalmente desde las empresas, se normalizada a través de diversas estrategias que la ordenan y estabilizan. Todas estas pistas podrían probar su utilidad 
para cualquier estrategia anticorrupción exitosa que tratara de ponerse en práctica en el futuro en países como México.

\section{Bibliografía}

Ananad, Vikas, Blake Ashforth, Mahendra Joshi y Perry Martini (2004), "Business as usual: the acceptance and perpetuation of corruption in organizations", Academy of Management Executive, i8(2), pp. 39-55. DoI:10.5465/AME.2005.19417904.

Ainslie, George (200I), Breaking-down of will, Cambridge, Cambridge University Press

Arellano, David (2017), "Corrupción como proceso organizacional: comprendiendo la lógica de la desnormalización de la corrupción" en Contaduría y Administración, 62, pp. 810-826. DoI:I0.IOI6/j.cya.2016.01.005.

Arellano, David (2012), ¿Podemos reducir la corrupción en México? México, Centro de Investigación y Docencia Económicas.

Ashforth, Blake, Dennis Gioia, Sandra Robinson y Linda Treviño (2008), "Re-viewing organizational corruption". Academy of Management Review, 33(3), pp. 670-684. DoI:I0.5465/AMR.2008.32465714.

Ashforth, Blake y Vikas Ananad (2003), "The normalization of corruption in organizations", Research in Organizational Behavior, 25, pp. I-52. DoI:I0.IOI6/SoI9I-3085(03)2500I-2.

Bargh, John y Tanya Chartrand (1999), "The unbearable automaticity of being”, American Psychologist, 54(7), pp. 462-479. DOI* I0.1037/0003$066 \mathrm{X} .54 \cdot 7.462$.

Benson, Michael (1985). "Denying the guilty mind: accounting for involvement in a white-collar crime", Criminology, 23, pp. 583-607. DOI:IO.IIII/j.1745-9125.1985.tboo365.x

Berger, Peter y Thomas Luckman (1978), La construcción social de la realidad, Buenos Aires, Amorrortu.

Blundo, Giorgio, Jean Pierre Olivier y N. B. Arifari (2006), Everyday corruption and the state. Citizens and public officials in Africa, Reino Unido, Zed Books.

Bratsis, Peter (2003), "The construction of corruption, or rules of separation and illusions of purity in bourgeois societies", Social Text, 77(2I-24), pp. 9-33 DoI:IO.1215/01642472-2I-4_77-9. 
Bourdieu, Pierre (2014), Sobre el estado. Cursos en el College de France (1989-1992), Barcelona, Anagrama.

Chibnall, Steven y Peter Saunders (1977), "Worlds apart: notes on the social reality of corruption", The British Journal of Sociology, 28(2), pp. 138-I54. DOI* $10.2307 / 590207$.

Conklin, John (1977), Business crime in America, Englewood, Prentice-Hall.

Costas, Jana y Christopher Grey (2014), "Bringing secrecy into de the open: towards a theorization of the social processes of organizational secrecy", Organization Studies, 35(Io), pp. I423-1447. Dor: IO.1177/0170840613515470.

Cressey, Donald (1986), "Why managers commit fraud", Australian and New Zealand Journal of Criminology, 19, pp. 195-209. Dor: I0.II77/000486588601900402.

Felps, Will, Terence Mitchell y Eliza Byington (2006), "How, when, and why bad apples spoil the barrel: negative group members and dysfunctional groups", Research in Organizational Behavior, 27, pp. 175-222. DoI: I0.I016/Sor91-3085(06)27005-9.

Festinger, Leon (1957), A Theory of cognitive dissonance, Stanford, Stanford University Press.

Fisman, Raymond y Edward Miguel (2008), Economic gangsters, Princeton, Princeton University Press.

Fleming, Peter y Stelios Zyglidopoulos (2009), Charting corporate corruption, Northampton, Edward Elgar.

Gambetta, Diego (1993), The Sicilian mafia, Cambridge, Harvard University Press.

Geis, Gilbert y Robert Meier (1979), "The white-collar ofender", en H. Touch (ed.), Psychology of crime and criminal justice, Prospect Heights, Waveland Press.

Gigerenzer, Gerd (2002), Bounded rationality: the adaptive toolbox, Cambridge, Mit Press.

Goffman, Erwin (1983), La presentación de la persona en la vida cotidiana, Buenos Aires, Amorrortu.

Heywood, Paul (2015), Routledge Handbook of political corruption, Oxford, Routledge.

Houba, Harold y Wilko Bolt (2002), Credible threats in negotiations. A game-theoretic approach, Nueva York, Kluwer Academic Publishers. 
INEgi (20I4a), Encuesta Nacional de Victimización de Empresas (eNVE) 2014. Principales Resultados, México, INEgI.

INEGI (20I4b), Encuesta Nacional de Calidad e Impacto Gubernamental (ENCIG) 2013, Principales resultados, México, INegI.

INEGi (20I4c), Censo Económico 2014. Resultados definitivos, México, INEGI.

Johnston, Michael (2005), Syndromes of corruption, Cambridge, Cambridge University Press.

Kunda, Gideon (1992), Engineering culture, Filadelfia, Temple University.

Kish-Gephart, Jennifer, David Harrison y Linda Klebe Treviño (20I0), "Bad apples, bad cases, and bad barrels: meta-analytic evidence about sources of unethical decisions at work", Journal of Applied Psychology, 95(I), pp. I-I3. DOI: 10.1037/aooI7103.

Lerner, Jennifer y Philip Tetlock (1999), "Accounting for the effects of accountability", Psychological Bulletin, I25(2), pp. 255-275. DOI: 10.1037/0033$2909 \cdot 125 \cdot 2.255$.

Loyens, Kim (2014), "Rule bending by morally disengaged detectives: an ethnographic study", Police Practice and Research, I5(I), pp. 62-74. DoI: I0.1080/15614263.2013.77094I.

Noonan, John (1984), Bribes, The intellectual history of a moral idea, Berkeley, University of California Press.

Persson, Anna, Rothstein y Jan Teorell (2013), "Why Anticorruption Reforms Fail-Systemic Corruption as a Collective Action Problem", Governance, 26(3), pp. 449-47I. DoI: I0.IIII/j.I468-049I.20I2.01604.x.

Pinto, Jonathan, Carrie Leana y Frits Pil (2008), “Corrupt organizations or organizations of corrupt individuals? two types of organization-level corruption", Academy of Management Review, 33, pp. 685-709. DoI: I0.5465/AMR.2008.32465726.

Rothstein, Bo y Jan Teorell (2008), "What is quality of government? A theory of impartial governmental institutions" Governance 2I(2), pp. 165-190. DOI: I0.1III/j.I468-049I.2008.00391.x.

Secretaría de Desarrollo Económico (sEDECo) (2015), Reporte económico de la Ciudad de México, zer Trimestre, México, Gobierno de la Ciudad de México.

Scott, James (1969), "The analysis of corruption in developing countries", Comparative Studies in Society and History, II(3), pp. 315-34I. DOI: IO.IOI7/ Soolo417500005363. 
Sekerka, Leslie y Roxanne Zolin (2007), "Rule-Bending: Can Prudential Judgment Affect Rule Compliance and Values in the Workplace?", Public Integrity, 9(3), pp. 225-243. DOI:I0.2753/PINIO99-9922090302.

Simon, Herbert (1947), Administrative behavior, Chicago, Chicago University Press.

Sykes, Gresham y David Matza (1957),"Techniques of neutralization: a theory of delinquency", American Sociological Review, 22, pp. 664-670. Dor: I0.2307 / 2089195.

Transparencia Internacional (2017), Corruption perception Index 2016, <http:// www.tm.org.mx/wp-content/uploads/2017/or/mundial.png >.

Weber, Max (1964), Economía y sociedad, 2 ed., México, Fondo de Cultura Económica.

Zerubavel, Eviatar (2006), The elephant in the room. Silence and denial in everyday life, Oxford, Oxford University Press.

\section{Anexo I* Versión del cuestionario aplicado}

I. Con base en su experiencia o conocimiento: ¿las autoridades gubernamentales en su ciudad se pueden considerar corruptas?
a) $\quad \mathrm{Si}$
b) No
c) No sabe, no contestó

2. Durante 20I5, ¿̇algún servidor público o persona actuando en su nombre solicitó algún pago o soborno como condición para recibvir algún servicio, licencia, permiso?
a) $\quad \mathrm{Si}$
b) No soborno? en sobornos (en pesos)?
a) $\quad \mathrm{I}-5000$
b) $5000 \mathrm{I}-25000$
c) 2500I-I25000

c) No sabe, no quiso contestar

d) Si sí, ¿cuántas veces durante ese año de 2015 se solicitó algún tipo de

3. Aproximadamente ¿̨cuánto dinero pagó en sobornos la empresa el año de 2015 

d) I2500i o más
e) Sobornos no monetarios

4+ ¿Qué tipo de servicio estaba involucrado en el soborno?
a) Permisos
b) Licenciaas
c) Trámites
d) Otros

4a. Si el pago del soborno no era en dinero, ¿qué tipo de pago involucró?
a) Regalos materiales
b) Otros regalos
c) Otro tipo de pago

5. ¿A quién de la empresa el servidor público o su representante solicitó originalmente el soborno?
a) Empleado operativo
b) Gerente o ejecutivo
c) Director General
d) Otro

6. ¿Qué áreas de la empresa intervinieron en el proceso de pago del soborno?
a) Finanzas
b) Administración
c) Operativo
d) Otro

7+ ¿Qué categoría contable, si alguna, se utilize para procesar el soborno?
a) Gastos especiales
b) Otros gastos
c) No procesado contablemente, u otra categoría contable

8. En su experiencia ¿la solicitud de soborno la realizar el servidor público en su nombre o actúa como parte de una grupo o red de personas?
a) Lo hace en su nombre
b) Lo hace a nombre de un grupo o red 
c) No sabe, no contestó

9. ¿Para negociar los arreglos del pago y su cantidad y entrega, dónde usualmente se reúnen las partes?
a) En un lugar externo a la empresa como un restaurant o café
b) En la empresa
c) En la oficina gubernamental
d) En otro lado, no contestó

Io. Aproximadamente, ¿cuanta gente de la empresa es requerida a procesar el soborno para poder entregarlo?
a) $\quad \mathrm{I}-2$
b) 3-10
c) Io o más

II. De esas parsonas involucradas en el procesamiento del soborno, ¿cuánta sabe que está procesando exactamente eso, un soborno?
a) $\quad 100 \%$
b) $\quad 75 \%$
c) $50 \%$
d) Menos del $50 \%$

I2. En su experiencia, ¿el servidor público ha tratado alguna vez de cambiar las reglas que ya se habían negociado respecto del pago del soborno (por ejemplo, la cantidad o el tipo de pago)?
a) Sí
b) $\quad \mathrm{No}$
c) No sabe, no contestó

13. Si sí, ¿qué es lo que la empresa hizo la última vez que esto sucedió?
a) Aceptó los cambios solicitados
b) Renegoció los términos con el servidor público o su representante
c) No sabe, no contestó

14. En su opinión, ¿qué tan importante es para la empresa que los acuerdos establecidos con el servidor público sean respetados en el tiempo?
a) Muy importante 

b) Más o menos importante
c) No importante

I5. ¿Qué tipo de estrategias o acciones la empresa ha realizado para mantener los acuerdos originales de soborno con los servidores públicos?
a) Invitar a los servidores públicos a reuniones informales u otras activi- dades para construir una relación estable
b) Regalos o favores
c) Negociaciones fuertes o uso de amenazas (veladas o directas)
d) Otros

I6. La empresa alguna ve a demandando a un servidor público por soborno?
a) Sí
b) $\quad \mathrm{No}$
c) No sabe, no contestó

I7. Si sí, ¿por qué?
a) Problemas económicos de la empresa
b) Comportamiento abusivo del servidor público
c) Cambio de partido o grupo en el poder
d) Otro

I8. En su opinión, ¿la empresa ha cambiado debido aceptar pagar sobornos?
a) Para mal
b) Para bien
c) No ha cambiado
d) No sabe, no contestó

Resumen CURRICULAR DEL AUtOR

David Arellano Gault es doctor en Administración Pública por la Universidad de Colorado en Denver. Es miembro del Sistema Nacional de Investigadores Nivel 3. Profesor investigador del Centro de Investigación y Docencia Económicas (CIDE) desde 1986. Ha publicado diversos libros en editoriales como FCE y CIDE, y artículos en revistas arbitradas nacionales y extranjeras como Public Administration Review, Organization Studies y Revista Mexicana de Sociología. Su perspectiva principal está 
en introducir los elementos o variables organizacionales en diversos temas propios del sector público. Es miembro de diversos comités editoriales de revistas como Journal of Public Administration Research and Theory y American Review of Public Administration, entre varias otras. Ha sido editor y coeditor de revistas como Gestión y Política Pública y Organization Studies, de la cual es actualmente Senior Editor.

Citar como: Arellano Gault, David (2018), “Corrupción ¿̇calle de una sola vía? La internalización del soborno en empresas en México", Iztapalapa. Revista de Ciencias Sociales y Humanidades, núm. 84, año 39, enero-junio de 20I8, ISSN: 2007-9176; pp. 163-190. Disponible en <http://revistaiztapalapa. izt.uam.mx/index.php/izt/issue/archive $>$. 